\title{
Ferrimagnetic to paramagnetic transition and dielectric relaxation in $\mathrm{Ni}_{1-\mathrm{x}} \mathrm{Zn}_{\mathrm{x}} \mathrm{Fe}_{2} \mathrm{O}_{4}$ ferrites
}

\author{
S. S. Jadhav ${ }^{1}$, S. P. Jadhav ${ }^{2}$, R.S. Ramshetti ${ }^{3}$, S. R. Kamble , S. M. Patange $^{5 *}$ \\ ${ }^{I}$ D.S.M's Arts, Commerce and Science College, Department Physics, Jintur, 431509 (M.S.), India \\ ${ }^{2}$ Adarsh College, Department of Chemistry, Omerga (M.S.), India \\ ${ }^{3}$ Adarsh College, Department of Physics, Omerga (M.S.), India \\ ${ }^{4}$ S.M. Dnyandeo Mohekar College, Department of Physics, Kalam (M.S.), India \\ ${ }^{5}$ S.K. Mahavidyalaya, Department of Physics, Gunjoti (M.S.), India
}

\begin{abstract}
The spinel ferrite system $\mathrm{Ni}_{1-\mathrm{x}} \mathrm{Zn}_{\mathrm{x}} \mathrm{Fe}_{2} \mathrm{O}_{4}(\mathrm{x}=0-0.7, \Delta \mathrm{x}=0.1)$ was prepared by wet chemical co-precipitation method using sulfates of respective metal ions. The electrical resistivity and dielectric properties of the prepared samples have been studied as a function of temperature, frequency, and Zn content (x). The DC resistivity (@) was measured in the temperature range of 300 to $800 \mathrm{~K}$. The curves so plotted show two phases viz. ferrimagnetic and paramagnetic, wherein the transformation from former to later phase took place at the Curie temperature, $T_{C}$. The values of $T_{C}$ were found to decrease with the increase in $x$. The values of activation energy $(\Delta \mathrm{E}<0.2 \mathrm{eV})$ depicted the possibility of the hopping mechanism governing the conduction within the ferrites. The ferrite samples exhibited semiconducting behavior, where the electrical resistivity $(\rho)$ decreased with the increasing temperature. The dielectric constant $\left(\varepsilon^{\prime}\right)$ and dielectric loss $\left(\varepsilon^{\prime \prime}\right)$ were studied as a function of composition, temperature, and frequency.
\end{abstract}

Keywords: wet chemical co-precipitation method, DC resistivity, ferrimagnetic phase, paramagnetic phase, Curie temperature, dielectric properties.

\section{INTRODUCTION}

Ferrites, which are magnetic oxides, form a class of very good dielectric materials because of their high resistivity and low loss behavior. These properties have made them be utilized in technological applications over a wide range of frequencies. Ni-Zn ferrites are very important soft magnetic materials, having many applications in both low and high-frequency devices. Ni-Zn ferrites are also used in the fabrication of multilayer chip inductors (MLCI). Multilayer inductors are one of the groups of passive surface mounting devices (SMD), which is an important component for the latest electronic products such as cellular phones, notebook computers, video camera recorders, floppy drives, etc. [1,2]. Ni-Zn ferrites also play a useful role in many technological applications because of their high resistivity, low dielectric loss, high Curie temperature, and chemical stability [3-5]. These features make them a suitable material for numerous applications like RF coils, transformer cores, and antenna rods.

The analysis of Raman spectra of $\mathrm{Ni}-\mathrm{Zn}$ ferrites confirmed the displacement of $\mathrm{Fe}^{2+}$ ions due to substitution of $\mathrm{Zn}^{2+}$ ions; the displacement of ions leads to interesting physical properties of the ferrite [6]. Recently Ni-Zn ferrites possessing interesting optical and electrical properties suitable for sensor applications were reported by Sankaranarayanan et al. [7]. A long period for dielectric

*smpatange@rediffmail.com

Dhttps://orcid.org/0000-0003-3970-5537 relaxation was reported for bulk Ni-Zn ferrite suggesting the ferrite as a promising candidate for future spintronic devices [8]. Analysis of frequency-dependent dielectric properties of Ni-Zn ferrites shows the complex dielectric behavior of the ferrite due to the presence of different relaxation mechanisms within the $20 \mathrm{~Hz}$ to $20 \mathrm{MHz}$ frequency band [9]. The Curie temperature $\left(\mathrm{T}_{\mathrm{C}}\right)$ study of Ni-Zn ferrites reports that the ferrites can be configured for a suitable value of $T_{C}$ by varying the composition of the constituent elements; the ferrites possess high thermal stability owing to higher $\mathrm{T}_{\mathrm{C}}$ [10]. The cation distribution study of Ni-Zn ferrites reported that the cations of the compositional elements are not specific about their occupancy at a sub-lattice site, suggesting the creation of a mixed spinel phase within the ferrite [11]. Due to this importance, we are presenting here a systematic study on the DC electrical resistivity and dielectric properties of nanocrystalline Ni-Zn ferrites prepared by a chemical coprecipitation method.

\section{EXPERIMENTAL}

The spinel ferrite system $\mathrm{Ni}_{1-\mathrm{x}} \mathrm{Zn}_{\mathrm{x}} \mathrm{Fe}_{2} \mathrm{O}_{4}$ with a variable composition ( $\mathrm{x}=0.0-0.7, \Delta \mathrm{x}=0.1$ ) was prepared by air oxidation of an aqueous suspension containing $\mathrm{Ni}^{2+}$, $\mathrm{Zn}^{2+}$ and $\mathrm{Fe}^{3+}$ cations in proper proportions. The starting solutions were prepared by mixing a $50 \mathrm{~mL}$ aqueous solution of $\mathrm{FeSO}_{4} .7 \mathrm{H}_{2} \mathrm{O}, \mathrm{NiSO}_{4} \cdot 7 \mathrm{H}_{2} \mathrm{O}$, and $\mathrm{ZnSO}_{4} .7 \mathrm{H}_{2} \mathrm{O}$ in stoichiometric proportion. A $2 \mathrm{M}$ solution of $\mathrm{NaOH}$ was prepared as a precipitant and added to the aqueous solution of sulfates to obtain the suspension $(\mathrm{pH}=11)$ containing 
dark-colored intermediate precipitant. The suspension was then heated at a constant temperature of $60^{\circ} \mathrm{C}$. The process of continuous stirring while heating, bubbled the oxygen into the suspension, promoting the oxidation reaction. The reaction continued till it yielded the browncolored precipitate of the ferrite. The ferrite precipitate was filtered and washed several times by distilled water. The wet samples of the $\mathrm{Ni}-\mathrm{Zn}$ system were annealed at $600{ }^{\circ} \mathrm{C}$ for $12 \mathrm{~h}$. Details of this method of preparation are discussed in our previous publication [12]. DC electrical resistivity measurements of all the samples were carried out on a discshaped pellet of $10 \mathrm{~mm}$ diameter and $2 \mathrm{~mm}$ thickness. The measurements were carried out in the range of 300 to $800 \mathrm{~K}$ using the two-probe method. The dielectric constant $\left(\varepsilon^{\prime}\right)$ and dielectric loss $(\varepsilon ")$ were measured as a function of frequency and temperature using an LCR-Q meter (HP 4284).

\section{RESULTS AND DISCUSSION}

The Rietveld refined XRD (X-ray diffraction) patterns of the Ni-Zn ferrite system for the typical samples with $\mathrm{x}=$ 0.1, 0.3, and 0.5 are shown in Fig. 1. All the peaks were indexed according to Bragg's law [13], and those matched well with the peaks of $\mathrm{NiFe}_{2} \mathrm{O}_{4}$ in JCPDS file 44-1485 [14]. The XRD analysis thus confirmed the formation of the single spinel phase in the ferrite crystal with space group $\mathrm{Fd} 3 \mathrm{~m}$. The amounts of $\mathrm{O}, \mathrm{Fe}, \mathrm{Ni}$, and $\mathrm{Zn}$ at tetrahedral and octahedral sites were quantified by using the Rietveld method. The obtained results of occupancy of the atoms are listed in Table I. It was clear that $\mathrm{Zn}$ had strong occupancy at the tetrahedral site only. However, $\mathrm{Fe}$ and $\mathrm{Ni}$ did not exhibit preference to a particular sub-lattice site. The refined XRD patterns were used for calculation of the particle size (t) from Scherer's relation:

$$
\mathrm{t}=\frac{0.9 \lambda}{\beta \cos \theta}
$$

where, $\lambda$ is the wavelength of X-ray $(=1.54 \AA), \beta$ the full width at half maximum of the (311) peak, and $\theta$ the angle of diffraction. The obtained values are listed in Table II. The magnitude of $\mathrm{t}$ less than $100 \mathrm{~nm}$ indicated the formation of the ferrite nanoparticles.

DC electrical resistivity measurements were performed on the disc-shaped pellets by varying the temperature from

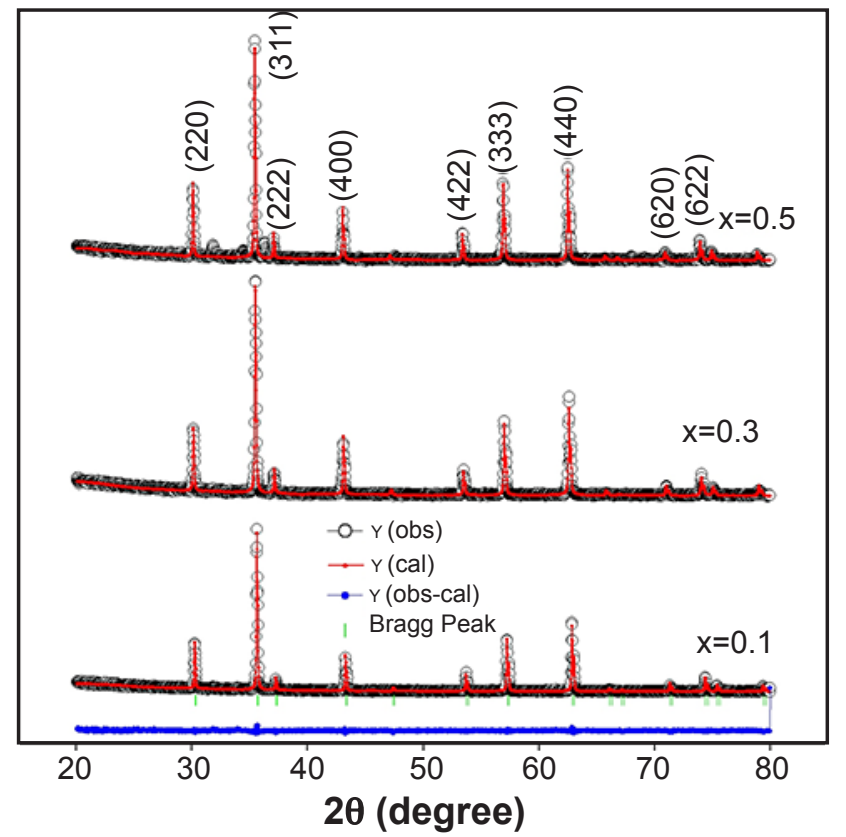

Figure 1: XRD patterns of the typical Ni-Zn ferrites with $\mathrm{x}=0.1$, 0.3 and 0.5 .

300 to $800 \mathrm{~K}$ for all the samples of the ferrite system. The variation of $\log (\mathrm{Q})$ with $10^{3} / \mathrm{T}$ for all the compositions is shown in Fig. 2. DC electrical resistivity of the present system was found to decrease with the increase of $\mathrm{Zn}$ concentration. The decrease in $\varrho$, on increasing $\mathrm{Zn}$ content, can be explained as follows. In the present $\mathrm{Ni}-\mathrm{Zn}$ ferrites, the $\mathrm{Zn}$ ions preferred the occupation of tetrahedral (A) sites and $\mathrm{Ni}^{2+}$ ions preferred the occupation of octahedral [B] sites, while $\mathrm{Fe}^{3+}$ ions partially occupied the (A) and [B] sites. On increasing $\mathrm{Zn}^{2+}$ ion substitution at (A) sites, $\mathrm{Ni}^{2+}$ ion concentration at $[\mathrm{B}]$ sites decreased. This led to the migration of some $\mathrm{Fe}^{3+}$ ions from (A) site to the [B] site to substitute the reduction in $\mathrm{Ni}^{2+}$ ion concentration at $\mathrm{B}$-sites. As a result, the numbers of ferrous and ferric ions at the [B] site (which are responsible for electrical conduction in ferrites) increased due to which, the resistivity decreased with $\mathrm{Zn}$ substitution in the ferrites. A similar trend, but with a different magnitude of resistivity has been reported by Ghazanfar et al. [15].

The decrease in resistivity of the ferrites with increasing temperature depicted in Fig. 2 confirmed that the ferrites

Table I - Values of occupancy of constituent elements of $\mathrm{Ni}_{x} \mathrm{Zn}_{1-x} \mathrm{Fe}_{2} \mathrm{O}_{4}$. The atoms showing occupancy at the octahedral site are shown by square brackets and those at the tetrahedral site are shown by parentheses.

\begin{tabular}{ccccccccc}
\hline Atom & $\mathrm{x}=0.0$ & $\mathrm{x}=0.1$ & $\mathrm{x}=0.2$ & $\mathrm{x}=0.3$ & $\mathrm{x}=0.4$ & $\mathrm{x}=0.5$ & $\mathrm{x}=0.6$ & $\mathrm{x}=0.7$ \\
\hline $\mathrm{O}$ & 4.00 & 4.00 & 4.00 & 4.00 & 4.00 & 4.00 & 4.00 & 4.00 \\
{$[\mathrm{Ni}]$} & 0.98 & 0.87 & 0.76 & 0.65 & 0.54 & 0.43 & 0.32 & 0.21 \\
{$[\mathrm{Fe}]$} & 1.02 & 1.13 & 1.24 & 1.35 & 1.46 & 1.57 & 1.68 & 1.79 \\
$(\mathrm{Ni})$ & 0.02 & 0.03 & 0.04 & 0.05 & 0.06 & 0.07 & 0.08 & 0.09 \\
$(\mathrm{Zn})$ & 0.00 & 0.10 & 0.20 & 0.30 & 0.40 & 0.50 & 0.60 & 0.70 \\
$(\mathrm{Fe})$ & 0.98 & 0.87 & 0.76 & 0.65 & 0.54 & 0.43 & 0.32 & 0.21 \\
\hline
\end{tabular}


Table II - Particle sizes (t), Curie temperatures $\left(\mathrm{T}_{\mathrm{C}}\right)$ and activation energies $\left(\mathrm{E}_{\mathrm{p}}, \mathrm{E}_{\mathrm{f}}\right.$ and $\left.\Delta \mathrm{E}\right)$ for $\mathrm{Ni}_{1-\mathrm{x}} \mathrm{Zn}_{\mathrm{x}} \mathrm{Fe}_{2} \mathrm{O}_{4}$.

\begin{tabular}{cccccc}
\hline \multirow{2}{*}{$\mathrm{x}$} & $\mathrm{t}(\mathrm{nm})( \pm 5 \mathrm{~nm})$ & $\mathrm{T}_{\mathrm{C}}(\mathrm{K})( \pm 1 \mathrm{~K})$ & \multicolumn{3}{c}{ Activation energy $(\mathrm{eV})( \pm 0.001 \mathrm{eV})$} \\
& & $\mathrm{E}_{\mathrm{p}}$ & $\mathrm{E}_{\mathrm{f}}$ & $\Delta \mathrm{E}=\mathrm{E}_{\mathrm{p}}-\mathrm{E}_{\mathrm{f}}$ \\
\hline 0.1 & 90 & 862 & 0.763 & 0.587 & 0.176 \\
0.2 & 85 & 840 & 0.638 & 0.506 & 0.132 \\
0.3 & 79 & 820 & 0.625 & 0.497 & 0.128 \\
0.4 & 91 & 797 & 0.594 & 0.478 & 0.116 \\
0.5 & 85 & 733 & 0.555 & 0.464 & 0.091 \\
0.6 & 90 & 704 & 0.545 & 0.451 & 0.094 \\
0.7 & 87 & 671 & 0.531 & 0.443 & 0.088 \\
\hline
\end{tabular}

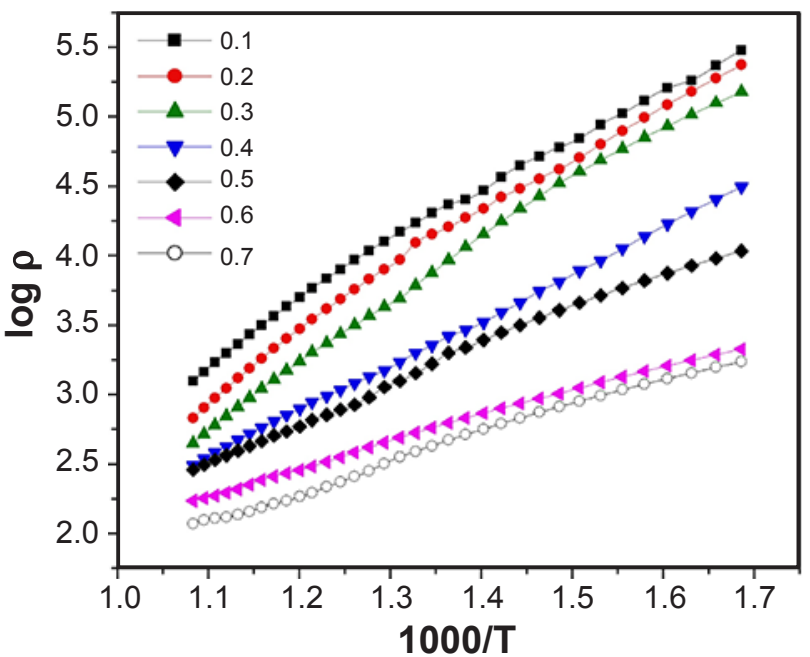

Figure 2: Variation of DC resistivity (@) with reciprocal temperature $(1 / \mathrm{T})$.

under investigation had semiconducting behavior. The variation of resistivity with the increase in temperature showed a perceptible kink near the transition temperature in all the cases. This transition temperature at which the slope of logarithm of resistivity versus reciprocal of temperature curve changed was identified as the Curie temperature $\left(\mathrm{T}_{\mathrm{C}}\right)$. The derivatives of the curves (d@/dT) are exhibited in Fig. 3 in which the $1 / T_{C}$ values are clearly indicated by the arrows over the curve at the Curie point of the ferrite. These values of $\mathrm{T}_{\mathrm{C}}$ obtained from curves of $\log (\mathrm{Q}) \mathrm{vs} .10^{3} / \mathrm{T}$ are listed in Table II. It was clear that the Curie temperature decreased with the increase in $\mathrm{x}$. This was attributed to the decreasing A-B interaction resulting from the replacement of magnetic $\mathrm{Fe}^{3+}$ ions by non-magnetic $\mathrm{Zn}^{2+}$ ions. It can be noted from Figs. 2 and 3 that at the values of $T_{C}$, a kink in each phase occurred at the Curie point of the corresponding ferrite. Similar transitions near Curie temperature have also been observed in other ferrite systems [12]. It has been shown theoretically that on passing through the Curie point, a change must occur in the gradient of the straight line, and the magnitude of the effect depends upon the exchange interaction. The experimental observation of transition near the Curie point is thus in conformity with the theory developed by Irkin and Turov [16]. The values of activation energies in the ferrimagnetic and paramagnetic regions were calculated from the slopes of $\log (\varrho)$ vs. $1 / T$ plots. The variation of resistivity with temperature obeys the Arrhenius relation:

$$
\rho=\rho_{0} \exp \left[\frac{\Delta \mathrm{E}}{\mathrm{k} \cdot \mathrm{T}}\right]
$$

where $\Delta \mathrm{E}$ is the activation energy in $\mathrm{eV}, \mathrm{\varrho}_{0}$ is the resistivity at room temperature, $\mathrm{k}_{\mathrm{B}}$ is the Boltzmann constant and $\mathrm{T}$ is the absolute temperature. The activation energy values corresponding to the two regions (before and after Curie temperature) were estimated using Eq. B for each sample and are presented in Table II. The decrease in the activation energy may be attributed to the creation of a small number of oxygen vacancies. It may also be justified due to the decrease in resistivity with the increase in $\mathrm{Zn}$ concentration because the activation energy behaves in the same way as that of the DC electrical resistivity [17]. In the present $\mathrm{Ni}-\mathrm{Zn}$ ferrite series, the preference of $\mathrm{Ni}^{2+}$ ions at octahedral [B] site and $\mathrm{Fe}^{3+}$ ions on tetrahedral (A) site as well as octahedral [B] site favored the following exchange interaction: $\mathrm{Ni}^{2+}+\mathrm{Fe}^{3+} \leftrightarrow \mathrm{Ni}^{3+}+\mathrm{Fe}^{2+}$. Thus, the conduction mechanism for the n-type semiconductor is predominantly due to the hopping of electrons from $\mathrm{Fe}^{2+}$ to $\mathrm{Fe}^{3+}$, while the conduction mechanism for the p-type semiconductor is due to the transfer from $\mathrm{Ni}^{3+}$ to $\mathrm{Ni}^{2+}$ [18]. It is clear from Table II that the activation energy for conduction in the ferrimagnetic region $\left(\mathrm{E}_{\mathrm{f}}\right)$ was lower than that in the paramagnetic region $\left(\mathrm{E}_{\mathrm{p}}\right)$. The activation energy $(\Delta \mathrm{E})$ of the ferrite sample is given by Eq. C. In the present series, the activation energy $(\Delta \mathrm{E})$ was found to be below $0.2 \mathrm{eV}$, therefore, the conduction in these ferrites might be due to the hopping of electrons.

$$
\Delta \mathrm{E}=\mathrm{E}_{\mathrm{p}}-\mathrm{E}_{\mathrm{f}}
$$

The variation of dielectric constant $\left(\varepsilon^{\prime}\right)$ with temperature is shown in Fig. 4a for all the values of $x$. It was evident that $\varepsilon$ ' increased with the increase in temperature. Similar nature was observed in different cases for other ferrites [1, $19,20]$. Fig. 4b shows the variation of dielectric loss ( $\varepsilon ")$ as a function of temperature. It was evident that $\varepsilon$ " also increased with the increase in temperature. The variation of 

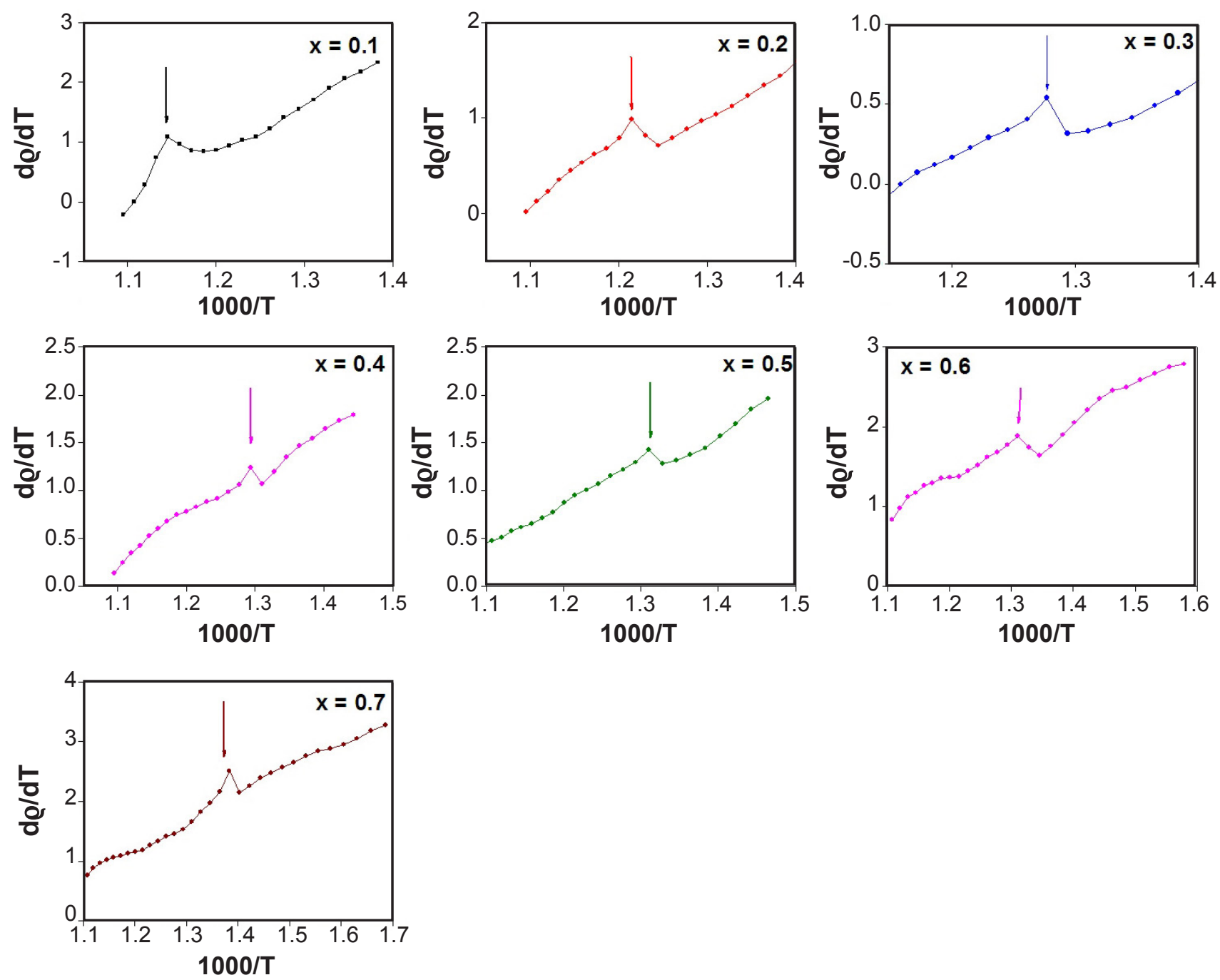

Figure 3: Variation of derivative of DC resistivity (d@/dT) with reciprocal of temperature (1/T).
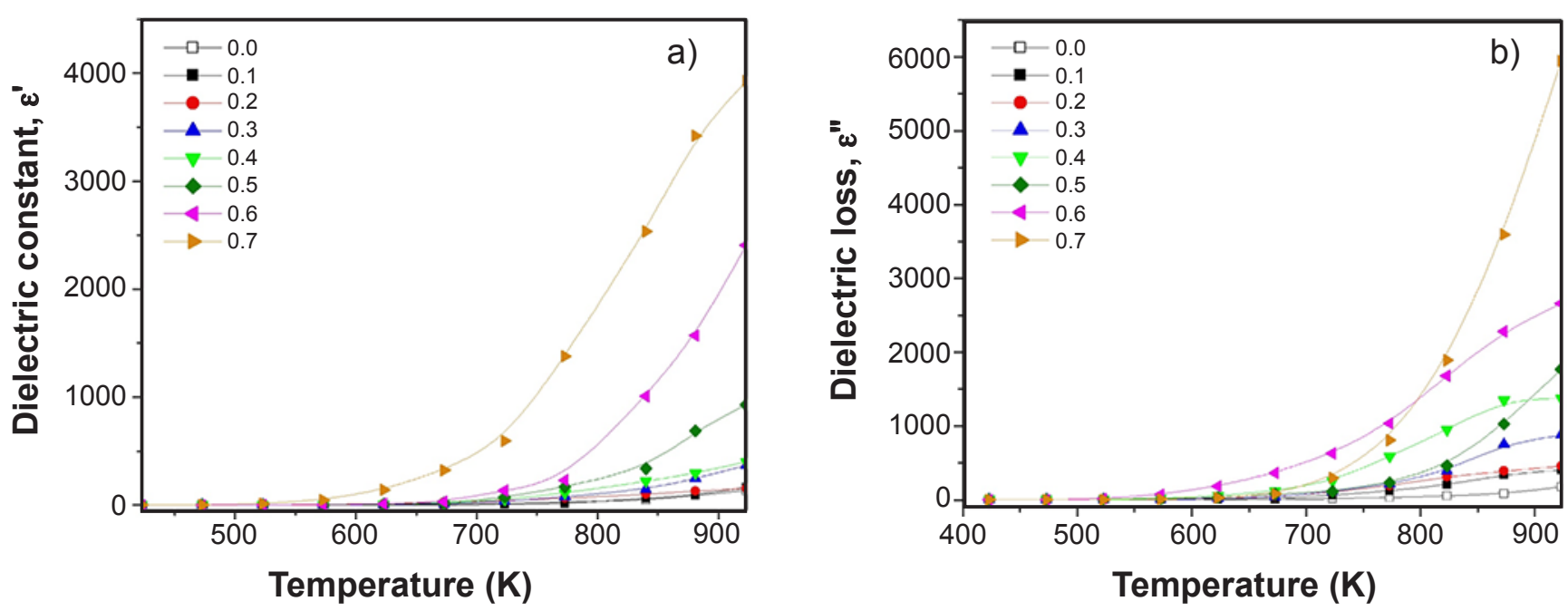

Figure 4: Variation with temperature (T) of: a) dielectric constant ( $\left.\varepsilon^{\prime}\right)$; and b) dielectric loss ( $\left.\varepsilon^{\prime \prime}\right)$.

$\varepsilon$ ' and $\varepsilon "$ can be explained on the basis of the polarization process. According to Rabkin and Novikova [21], the process of dielectric polarization in ferrites takes place through a mechanism similar to the conduction process by electron exchange $\mathrm{Fe}^{2+} \leftrightarrow \mathrm{Fe}^{3+}$ and $\mathrm{Ni}^{2+} \leftrightarrow \mathrm{Ni}^{3+}$ from which one obtains local displacement of the electrons in the direction of electrical field; these displacements determine the polarization. Both types of carriers i.e. ' $n$ ' and ' $p$ ' 

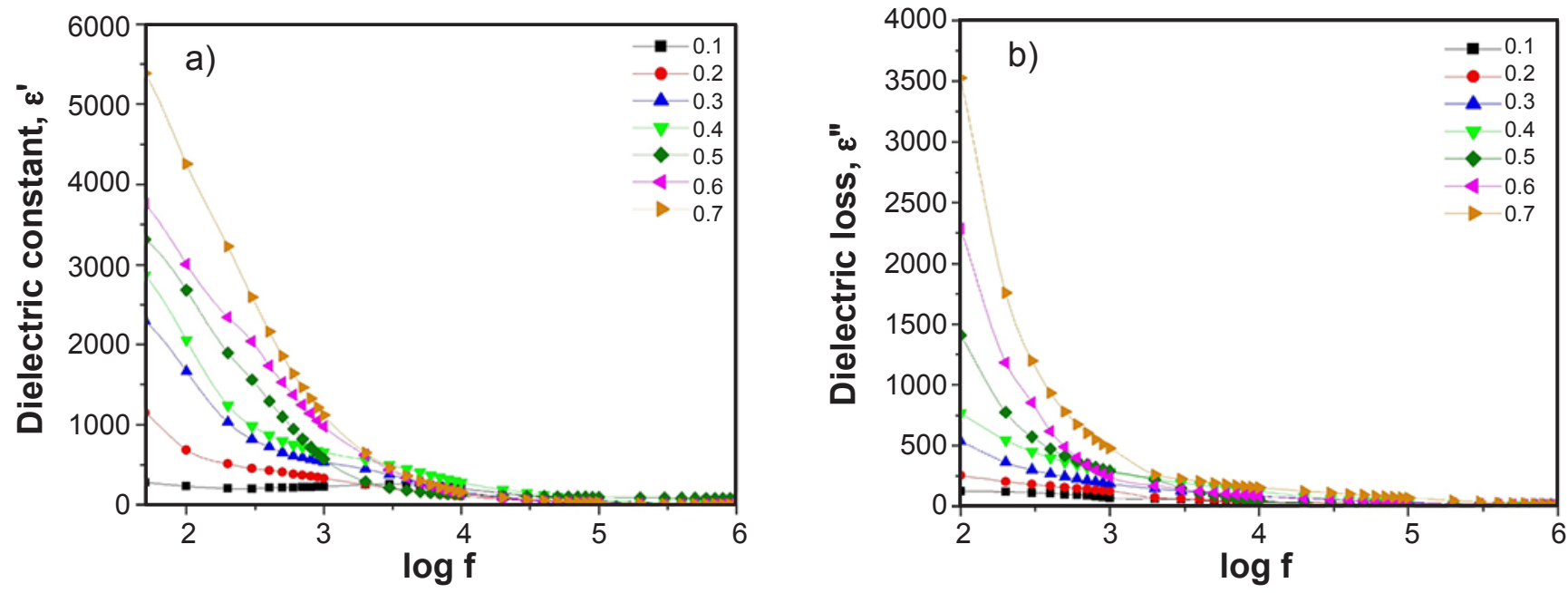

Figure 5: Variation with frequency (f) of: a) dielectric constant ( $\left(\varepsilon^{\prime}\right)$; and b) dielectric loss $\left(\varepsilon^{\prime \prime}\right)$.

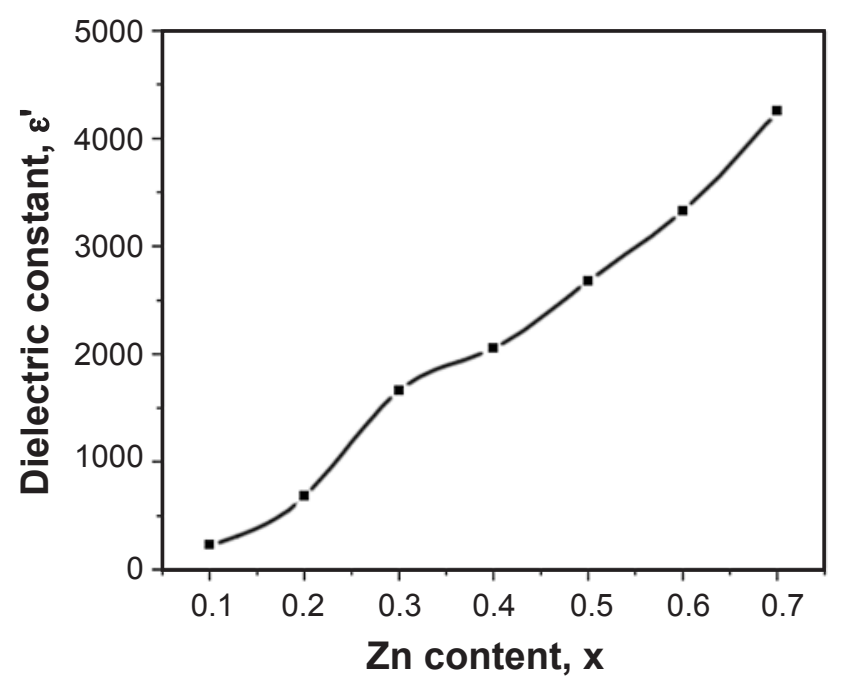

Figure 6: Variation of dielectric constant ( $\left.\varepsilon^{\prime}\right)$ with $\mathrm{Zn}$ content $(\mathrm{x})$ at a constant frequency $(100 \mathrm{~Hz})$.

contribute to the polarization and their contribution depends on the temperature. Hence, the influence of temperature on the electronic exchange $\mathrm{Fe}^{2+} \leftrightarrow \mathrm{Fe}^{3+}$ and $\mathrm{Ni}^{2+} \Theta \mathrm{Ni}^{3+}$ is more pronounced than on the displacement of peak carriers, due to which $\varepsilon^{\prime}$ and $\varepsilon^{\prime \prime}$ increase rapidly with the increase in temperature.

Fig. 5a shows the variation of dielectric constant with frequency for all the samples. It was seen that the dielectric constant $\varepsilon$ ' decreased with the increase in frequency. The behavior of frequency dependence of $\varepsilon^{\prime}$ for $\mathrm{Ni}_{1-x} \mathrm{Zn}_{x} \mathrm{Fe}_{2} \mathrm{O}_{4}$ is in good agreement with those of the $\mathrm{Y}^{3+}$ substituted $\mathrm{Mg}$ - Zn ferrites [22], for which $\varepsilon$ ' continuously decreases with increasing frequency. More dielectric dispersion is observed in the lowfrequency region, which may be due to the Maxwell-Wagner interfacial type of polarization [23, 24], which is in good agreement with Koop's phenomenological theory [25]. The decrease in polarization with the increase in frequency may be due to the fact that, beyond a certain frequency of the electric field, the electronic exchange between $\mathrm{Fe}^{2+}$ and $\mathrm{Fe}^{3+}$ cannot follow the alternating field; therefore, the dielectric constant decreases with increasing frequency. The variation of dielectric loss ( $\varepsilon$ ") with frequency at room temperature is depicted in Fig. 5b for the ferrite samples. All the samples showed a normal dielectric behavior with the frequency where the parameter $\varepsilon$ " also decreased exponentially with the increase of frequency. A typical plot showing the variation of dielectric constant with $\mathrm{x}$ is shown in Fig. 6. It shows that $\varepsilon$ ' increases with increasing $\mathrm{Zn}$ content. This may indicate the possibility of a decrease in the hopping interaction with the increasing addition of $\mathrm{Zn}^{2+}$ ions. In the present $\mathrm{Ni}-\mathrm{Zn}$ ferrites, $\mathrm{Zn}^{2+}$ ions occupy tetrahedral (A) sites whereas $\mathrm{Ni}^{2+}$ ions prefer to go to the octahedral $[\mathrm{B}]$ sites. The Fe ions, which exist in $2+$ as well as in $3+$ states, occupy both (A) and [B] sites. When $\mathrm{Zn}^{2+}$ is added in place of $\mathrm{Ni}^{2+}$ ions, some of the $\mathrm{Fe}^{3+}$ ions are converted to $\mathrm{Fe}^{2+}$ ions to maintain charge neutrality. As a result, hopping between $\mathrm{Fe}^{3+}$ and $\mathrm{Fe}^{2+}$ ions increases leading to a decrease in grain resistance. This increases the probability of electrons reaching the grain boundary. Consequently, polarization and dielectric constant increase [26].

\section{CONCLUSIONS}

The Zn-substituted $\mathrm{NiFe}_{2} \mathrm{O}_{4}$ nanoparticles were successfully prepared by a wet chemical co-precipitation method. The particle sizes calculated from the refined XRD patterns varied from 79 to $90 \mathrm{~nm}$. The presence of a single spinel phase in the ferrites was confirmed from the Rietveld refinement of the XRD pattern. The occupancy of the elements obtained from the Rietveld method suggested that $\mathrm{Zn}$ showed a strong preference for the tetrahedral site while $\mathrm{Ni}$ and $\mathrm{Fe}$ showed their occupancy to both the sublattice (tetrahedral and octahedral) sites. DC electrical resistivity study showed the presence of ferrimagnetic and paramagnetic phases for the two different temperature regions in Ni-Zn ferrite. The values of Curie temperature of the ferrite $\left(\mathrm{Ni}_{1-\mathrm{x}} \mathrm{Zn}_{x} \mathrm{Fe}_{2} \mathrm{O}_{4}\right)$ samples decreased from 862 to $671 \mathrm{~K}$ with the increase in $\mathrm{x}$, which may be due to a decrease in strength of A-B interaction with increasing $\mathrm{x}$. 
The values of activation energy were less than $0.2 \mathrm{eV}$, which indicated that the hopping mechanism was governing the conduction in the ferrites. The dielectric polarization in the ferrites was strongly influenced by temperature, wherein more dispersion in values of dielectric constant $(\varepsilon$ ') occurred for temperatures $>700 \mathrm{~K}$. The dielectric dispersion at low frequencies $(<10 \mathrm{kHz})$ indicated the presence of MaxwellWagner interfacial polarization in the ferrite system. Increasing the concentration of $\mathrm{Zn}^{2+}$ in the $\mathrm{Ni}-\mathrm{Zn}$ ferrite system increased its dielectric constant. The dielectric study suggested the suitability of $\mathrm{Ni}-\mathrm{Zn}$ ferrite synthesized by wet chemical co-precipitation method for high-frequency applications.

\section{REFERENCES}

[1] A. Bhaskar, B.R. Kanth, S.R. Murthy, J. Magn. Magn. Mater. 283 (2004) 109.

[2] M.P. Reddy, W. Madhuri, G. Balakrishnaiah, N.R. Reddy, K.V. Siva Kumar, V.R.K. Murthy, R.R. Reddy, Current Appl. Phys. 119 (2011) 191.

[3] A. Verma, O.P. Thakur, C. Prakash, T.C. Goel, R.G. Mendiratta, Mater. Sci. Eng. B 116 (2005) 1.

[4] S.A. Saafan, T.M. Meaz, E.H. El-Ghazzawy, M.K. ElNimr, M.M. Ayad, M. Bakr, J. Magn. Magn. Mater. 322 (2010) 2369.

[5] P.I. Slick, "Ferromagnetic materials", W.P. Wohlforth (Ed.), 2, North-Holland, Amsterdam (1980) 189.

[6] C.O. Ehi-Eromosele, B.I. Ita, E.E.J. Iweala, S.A. Adalikwu, P.A.L. Anawe, Bull. Mater. Sci. 38 (2015) 1.

[7] R. Sankaranarayanan, S. Shailajha, M.S.K. Mubina, C.P. Anilkumar, J. Mater. Sci. Mater. Electron. 31 (2020) 11833. [8] M.A. Ali, M.N.I. Khan, F.U.Z. Chowdhury, S. Akhter, M.M. Uddin, J. Sci. Res. 7 (2015) 65.

[9] A. Safari, K. Gheisari, M. Farbod, J. Magn. Magn. Mater. 488 (2019) 165369.
[10] M.M. Kothawale, R.B. Tangsali, S.S. Meena, N.K. Prasad, A. Gangwar, J. Supercond. Nov. Magn. 32 (2019) 2141.

[11] C.A.P. Gomez, C.A.B. Meneses, A. Matute, Mater. Sci. Eng. B 236-237 (2018) 48.

[12] S.M. Patange, S.E. Shirsath, B.G. Toksha, S.S. Jadhav, K.M. Jadhav, J. App. Phys. 106 (2009) 23914.

[13] B.D. Cullity, Elements of X-ray diffraction, Addison Wesley Publ., Massachusetts (1956) 84.

[14] S.K. Gore, S.S. Jadhav, U.B. Tumberphale, S.M. Shaikh, M. Naushad, R.S. Mane, Solid State Sci. 74 (2017) 88.

[15] U. Ghazanfar, S.A. Siddiqi, G. Abbas, Mater. Sci. Eng. B 118 (2005) 132.

[16] Y.P. Irkin, E.A. Turov, Soviet Phys. J. Exper. Phys. 33 (1957) 673.

[17] M. El-Shabasy, J. Magn. Magn. Mater. 172 (1997) 188. [18] L.G. Van Uitert, J. Chem. Phys. 23 (1955) 1883.

[19] S.S. Shinde, K.M. Jadhav, Mat. Lett. 37 (1998) 63.

[20] A.R. Shitre, V.B. Kawade, G.K. Bichile, K.M. Jadhav, Mater. Lett. 56 (2002) 188.

[21] R.I. Rabkin, Z.I. Novikova, in "Ferrites", Doklady Akad. Nauk SSSR, Minsk (1960) 146.

[22] M.A. Ali, M.N.I. Khan, F.U.Z. Chowdhury, M.M. Hossain, A.K. Hossain, M.A. Rashid, R. Nahar, S.M. Hoque, M.A. Matin, M.M. Uddin, J. Mater. Sci. Mater. Electron. 30 (2019) 13258.

[23] J.C. Maxwell, Electricity and magnetism, 1, Oxford Un. Press, Oxford (1929).

[24] M.D. Hossain, M.N.I. Khan, A. Nahar, M.A. Ali, M.A. Matin, S.M. Hoque, M.A. Hakim, A.T.M.K. Jamil, J. Magn. Magn. Mater. 497 (2020) 165978.

[25] C.G. Koops, Phys. Rev. 83 (1951) 121.

[26] A.K. Singh, T.C. Goel, R.G. Mendiratta, J. Appl. Phys. 91 (2002) 6626.

(Rec. 06/06/2020, Rev. 11/09/2020, 09/11/2020, Ac. $13 / 11 / 2020)$ 